\title{
Propagation Effects in Photonic Liquid Crystal Fibers with a Complex Structure
}

\author{
M.M. Tefelska ${ }^{a, *}$, M.S. Chychlowski ${ }^{a}$, T.R. Woliński ${ }^{a}$, R. DĄBrowski ${ }^{b}$, \\ W. REJMER ${ }^{b}$ AND J. WÓJCIK ${ }^{c}$ \\ ${ }^{a}$ Faculty of Physics, Warsaw University of Technology, Koszykowa 75, 00-662 Warsaw, Poland \\ ${ }^{b}$ Military University of Technology, Warsaw, Poland \\ ${ }^{c}$ Maria Curie Skłodowska University, Lublin, Poland
}

\begin{abstract}
Chiral nematic liquid crystals are very interesting materials to infiltrate photonic crystal fibers since they are characterized by unique optical properties such as selective Bragg reflection, circular dichroism, and optical activity. In this paper the latest experimental results of the photonic crystal fibers filled with new chiral nematic liquid crystals are presented. Spectral properties of the light propagating in the photonic liquid crystal fibers have been measured and analyzed.
\end{abstract}

PACS numbers: 42.81.Gs, 78.20.Bh, 42.79.Kr

\section{Introduction}

Photonic liquid crystal fibers (PLCFs) with a complex structure are solid-core photonic crystal fibers infiltrated with specific nematic liquid crystals (LCs) doped with optically active dopants (OAD). There are two general mechanisms of light propagating in photonic crystal fibers: the modified total internal reflection (mTIR) and the photonic band gap (PBG) mechanism. If the refractive index of the filling liquid crystal is lower than the refractive index of the photonic crystal fiber (PCF) glass, all wavelengths are guided within the core by the mTIR [1]. However if the refractive index of the LC is higher than the refractive index of the silica glass, only selected wavelengths can be guided due to the PBG phenomenon.

Light propagation can be easily modified in photonic crystal fibers either by a special fiber structure design or by filling these microstructured fibers with various materials of particular properties [2-9].

LCs are particularly interesting substances to infiltrate PCFs, since their optical properties strongly depend on thermal, electric, magnetic, and optic fields. Photonic crystal fibers can be also filled with nematic liquid crystals doped with chiral compounds - optically active dopants (OADs) e.g. cholesteric dopants, azo-compounds, dyes that can modify switching and sensing possibilities of the PLCFs [9]. Chiral nematic liquid crystals (ChNLCs) [10] seem to be novel and interesting materials to infiltrate PCFs. The chiral nematic phase exhibits helicoidal twisting of the molecules oriented perpendicularly to a director, which is a dimensionless unit vector $\boldsymbol{n}$ and it represents a mean direction of preferred orientation of molecules in the neighborhood of the given point. Chirality induces a finite azimuthal twist from one layer to another, producing a helicoidal twisting of the

* corresponding author; e-mail: martef@if.pw.edu.pl molecular axis along the normal layer. Chiral nematic liquid crystals due to their spatial periodicity are characterized by unique optical properties such as selective Bragg reflection, circular dichroism, and optical activity. The selective Bragg reflection is a total reflection of the circularly polarized light (having the same handedness as the helical pitch of the chiral structure), occurring at the wavelength $\lambda=\bar{n} P$ where $\bar{n}$ is the mean refractive index and $P$ is the pitch (defined as a distance it takes for the director to rotate one full turn in the helix).

\section{Materials and experimental setup}

In this paper we present results of propagation studies in PCF filled with chiral nematic liquid crystalline mixtures marked as: PW500, PW600, PW700, PW1000 and PW1500 prepared at the Military University of Technology (Warsaw, Poland) which are characterized by significantly reduced temperature sensitivity of the selective Bragg reflection, see Fig. 1. The chiral nematic LCs used in the experiment were composed of alkylcyclohexyl bicyclohexylbenzene nitryles and isothiocyanates base mixture W-1816 and doped with optically active dopants (Fig. 2) formulated in concentrations presented in Table.

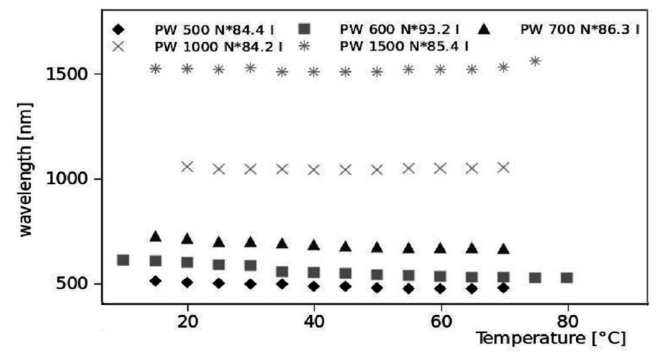

Fig. 1. The selective Bragg reflection for PW500, PW600, PW700, PW1000, PW1500 chiral nematic LC.

In the experiment two host solid-core PCFs: the 070124 PCF with 3 rings of air holes and the LMA 081120 


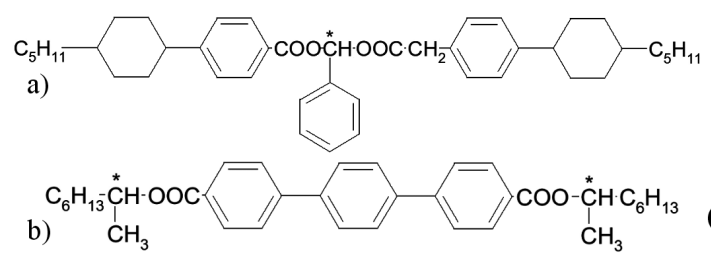

Fig. 2. Optically active dopants: (a) OAD 1 is right handed and with helicoidal twisting power HTP = $67.2 \mu \mathrm{m}^{-1}$, (b) OAD 2 is left handed and with HTP $=$ $30.7 \mu \mathrm{m}^{-1}$.

Parameters of ChNLC.

TABLE

\begin{tabular}{l|c|c|c|c|c}
\hline \hline $\begin{array}{c}\text { Chiral } \\
\text { nematics }\end{array}$ & $\begin{array}{c}\lambda_{\max } \\
{[\mathrm{nm}]}\end{array}$ & $\mathrm{N}^{*}$-Iso & $\begin{array}{c}\text { OAD1 } \\
{[\% \text { weight] }]}\end{array}$ & $\begin{array}{c}\text { OAD2 } \\
\text { [\%weight] }]\end{array}$ & $\begin{array}{c}\text { Pitch } \\
{[\mu \mathrm{m}]}\end{array}$ \\
\hline PW 500 & 500 & 84.4 & 10 & 0 & 0.31 \\
PW 600 & 600 & 83.2 & 9.4 & 0 & 0.40 \\
PW 700 & 700 & 86.3 & 5 & 0 & 0.43 \\
PW 1000 & 1000 & 84.2 & 1.3 & 0 & 0.60 \\
PW 1500 & 1500 & 85.4 & 0 & 0 & 0.95
\end{tabular}

PCF with 5 rings of holes (Fig. 3a,b), manufactured at the Maria Curie Skłodowska University (UMCS, Lublin, Poland) were used. The $070124 \mathrm{PCF}$ has a $3.9 \mu \mathrm{m}$ core diameter whereas the LMA 081120 PCF has the fiber cladding and core diameters of $\approx 130 \mu \mathrm{m}$ and $3.2 \mu \mathrm{m}$. As a light source a halogen lamp (Mikropack Halogen LightSource) was used. As a light intensity detector we used the HR4000 Ocean Optics spectrometer. Temperature was controlled by the Peltier module (Fig. 3) and the PCF was filled with the ChNLC by using capillary forces.

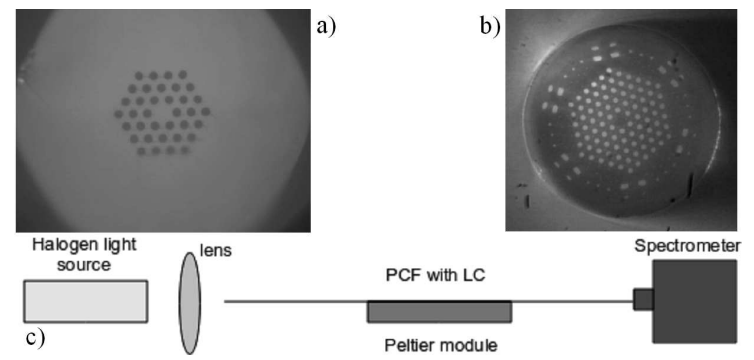

Fig. 3. (a) Cross-sections of the photonic crystal fiber with 3 (070124) and (b) 5 rings (LMA 081120) of holes, (c) experimental setup.

\section{Results}

The selective Bragg reflection was observed along the PCF filled with ChNLCs. Figure 4 presents the 070124 PCF filled either with PW500 or PW700 which selectively reflect light at the wavelengths 500 and $700 \mathrm{~nm}$, respectively. This phenomenon does not significantly affect the light propagation mechanism.

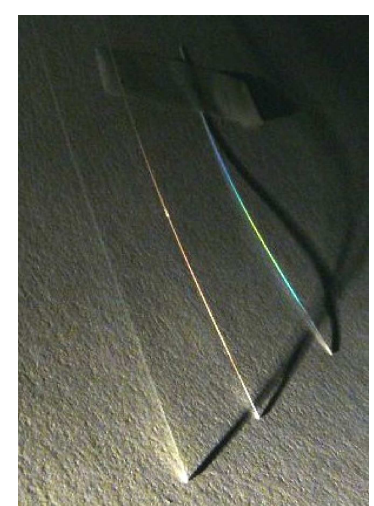

Fig. 4. Side views of the investigated PCFs (from left to right) 5-ring PCF (empty), PCF + PW700 ChNLC, PCF + PW500 ChNLC.

Since the mean refractive index of the ChNLC is higher than the effective refractive index of the fiber core, light can be guided due to the PBG propagation mechanism. We have also observed PBGs tuning under influence of temperature in the PCFs filled with a chiral nematic LC which is typical for PLCFs. Initially, the photonic crystal fiber with 3 rings of holes was filled by capillary forces with the PW500 LC in a $2.5 \mathrm{~cm}$ PCF section. We noticed that photonic band gaps were moving into shorter wavelengths when the temperature was increasing. The propagation for this sample was very good and the PBG tuning phenomenon was repeatable (Fig. 5).

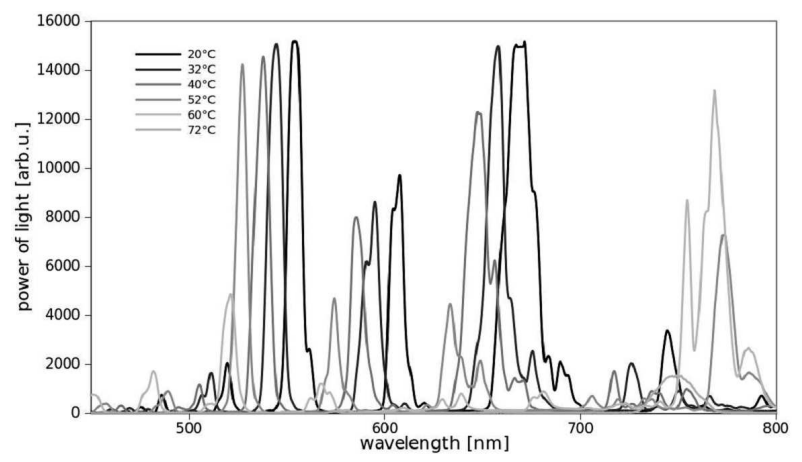

Fig. 5. Transmission spectra of PCF with 3 rings of holes filled with PW500 $(2.5 \mathrm{~cm})$.

It can be pointed out that during temperature changing the width of PBGs (for 500 to $700 \mathrm{~nm}$ range) was stable.

PCFs with 3 rings of holes filled with one of the ChNLCs: PW500, PW600, PW700, PW1000 or PW1500 were compared in Fig. 6. In case of the PCF with 3 rings of holes filled with PW500 ChNLC the highest output intensity was observed.

Comparing spectrum of the light for $\mathrm{PCF}$ with 3 rings of holes filled with 5CB LC nematic versus chiral nematic (Fig. 6) the power of light for PBGs is lower for 5CB than for chiral nematic LCs. 


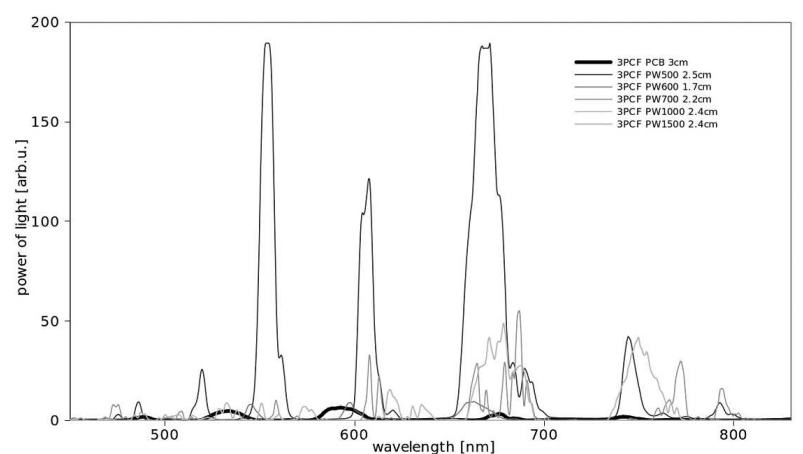

Fig. 6. Comparison of selective wavelengths in transmission spectra of PCFs with 3 rings of holes filled with PW500, PW600, PW700, PW1000, PW1500 and 5CB.

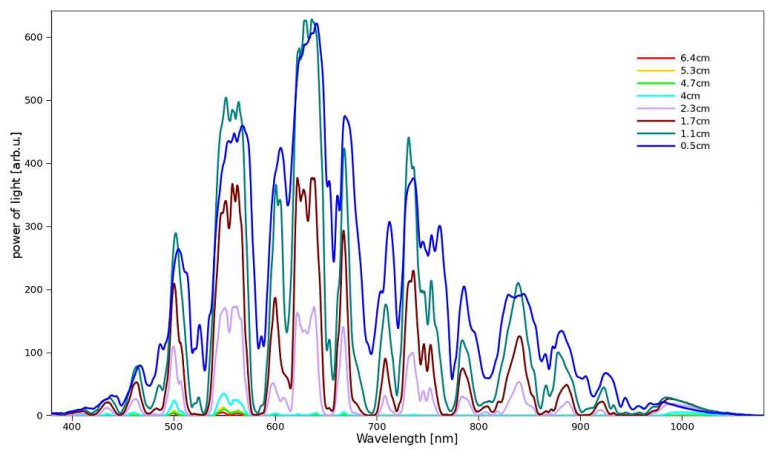

Fig. 7. The dependence of the PBG attenuation for different filled sections in PCF with 5 rings of holes filled with PW500 ChNLC.

For the PCF with 5 rings of holes filled with the PW500 PBGs tuning under the influence of temperature was observed. The section of LC infiltration was about $6.4 \mathrm{~cm}$ long. The PCF with the PW500 mixture was cut to smaller sections up to $0.5 \mathrm{~cm}$ to calculate the attenuation of light (Fig. 7). For the $6.4 \mathrm{~cm}$ long section with the PW500 filling, light attenuation was relatively high

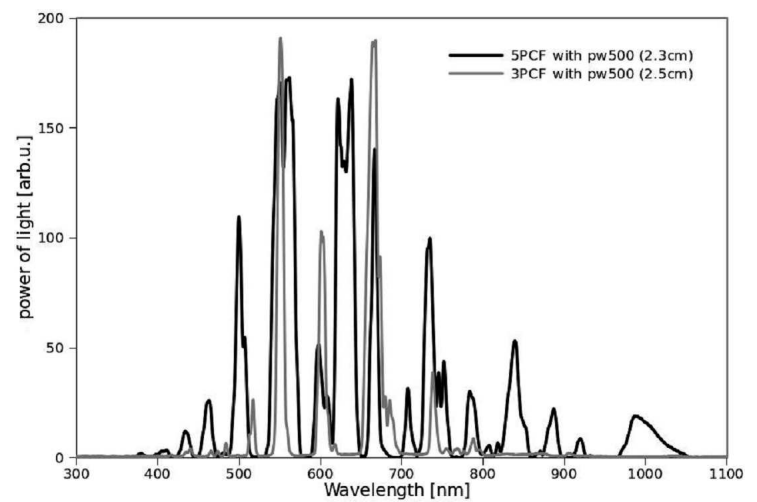

Fig. 8. Comparison of PCF with 3 and 5 rings of holes filled with PW500. and in particular for the wavelengths $435 \mathrm{~nm}$ and $630 \mathrm{~nm}$, residual propagation was observed. The longer infiltration lengths of the chiral nematic LC caused higher attenuation in the LC-infiltrated PCF.

In Fig. 8 we can see that PBGs for PCF with 3 and 5 rings of holes overlap although in case of $\mathrm{PCF}$ with 5 rings of holes the number of PBGs is higher.

\section{Conclusions}

The PCF with 3 rings of holes filled with the PW500 mixture demonstrates a possibility of optical filtering by temperature-induced tuning of the PBGs and attenuation of the fiber in a wide range of temperatures from at least $20^{\circ} \mathrm{C}$ to $60^{\circ} \mathrm{C}$ is very low. Consequently, this property may be used to propose a new idea of a fiber-optic filter that can be used in optical processing systems.

\section{Acknowledgments}

The work was supported by the Polish Ministry of Science and Education under the grant N517 056535, by the European Union in the framework of European Social Fund through the Warsaw University of Technology Development Programme and partially by the MISTRZ Programme of the Polish Science Foundation.

\section{References}

[1] T.T. Larsen, A. Bjarklev, D.S. Hermann, J. Broeng, Opt. Exp. 11, 2589 (2003).

[2] T.R. Woliński, A. Czapla, S. Ertman, M. Tefelska, A.W. Domański, E. Nowinowski-Kruszelnicki, R. Dąbrowski, Opt. Quant. Electron. 39, 1021 (2007).

[3] S. Ertman, A. Czapla, T.R. Woliński, T. Nasiłowski, H. Thienpont, E. Nowinowski-Kruszelnicki, R. Dąbrowski, Opto-Electron. Rev. 17, 150 (2009).

[4] F. Du, Y.Q. Lu, S.T. Wu, Appl. Phys. Lett. 85, 2181 (2004).

[5] T.R. Woliński, K. Szaniawska, K. Bondarczuk, P. Lesiak, A.W. Domański, R. Dąbrowski, E. Nowinowski-Kruszelnicki, J. Wójcik, Opto-Electron. Rev. 13, 177 (2005).

[6] D. Budaszewski, A.W. Domański, Acta Phys. Pol. A 116, 285 (2009).

[7] A.W. Domanski, P. Lesiak, K. Milenko, D. Budaszewski, M. Chychlowski, S. Ertman, M. Tefalska, T.R. Wolinski, K. Jedrzejewski, L. Lewandowski, W. Jasiewicz, J. Helsztynski, A. Boczkowska, Acta Phys. Pol. A 116, 294 (2009).

[8] D. Dorosz, M. Kochanowicz, J. Dorosz, Acta Phys. Pol. A 116, 298 (2009).

[9] T.T. Alkeskojld, L.A. Bjarklev, D.S. Hermann, Anawati, J. Broeng, J. Li, S.T. Wu, Opt. Exp. 12, 5857 (2004).

[10] H.-S. Kitzerow, B. Liu, F. Xu, P.P. Crooker, Phys. Rev. E 54, 568 (1996). 